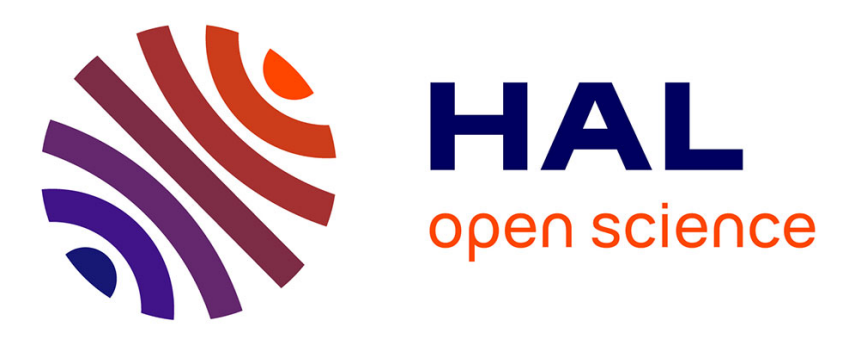

\title{
A new mechanism for increasing density peaking in tokamaks: Improvement of the inward particle pinch with edge $E \mathbf{X} B$ shearing
}

J. Garcia, H. Doerk, T. Görler

\section{- To cite this version:}

J. Garcia, H. Doerk, T. Görler. A new mechanism for increasing density peaking in tokamaks: Improvement of the inward particle pinch with edge EXB shearing. Theory of Fusion Plasmas joint Varenna -Lausanne International Workshop, Aug 2018, Varenne, Italy. 10.1088/1361-6587/ab31a4 . cea-02378868

\section{HAL Id: cea-02378868 https://hal-cea.archives-ouvertes.fr/cea-02378868}

Submitted on 25 Nov 2019

HAL is a multi-disciplinary open access archive for the deposit and dissemination of scientific research documents, whether they are published or not. The documents may come from teaching and research institutions in France or abroad, or from public or private research centers.
L'archive ouverte pluridisciplinaire HAL, est destinée au dépôt et à la diffusion de documents scientifiques de niveau recherche, publiés ou non, émanant des établissements d'enseignement et de recherche français ou étrangers, des laboratoires publics ou privés. 


\title{
A new mechanism for increasing density peaking in tokamaks: Improvement of the inward particle pinch with edge $E \times B$ shearing
}

\author{
J. Garcia, ${ }^{1}$ H. Doerk, ${ }^{2}$ T. Görler, ${ }^{2}$ and and JET Contributors ${ }^{3}$ \\ ${ }^{1}$ CEA, IRFM, F-13108 Saint-Paul-lez-Durance, France \\ ${ }^{2}$ Max-Planck-Institut für Plasmaphysik, Boltzmannstraße 2, D-85748 Garching, Germany \\ ${ }^{3}$ See the author list of Overview of the JET preparation for Deuterium-Tritium Operation \\ by E. Joffrin et al. to be published in Nuclear Fusion Special issue: overview and summary \\ reports from the 27th Fusion Energy Conference (Ahmedabad, India, 22-27 October 2018)
}

(Dated: July 4, 2019)

\begin{abstract}
Developing successful tokamak operation scenarios, as well as confident extrapolation of presentday knowledge requires a rigorous understanding of plasma turbulence, which largely determines the quality of the confinement. In particular, accurate particle transport predictions are essential due to the strong dependence of fusion power or bootstrap current on the particle density details. Here, gyrokinetic turbulence simulations are performed with physics inputs taken from a JET power scan, for which a relatively weak degradation of energy confinement and a significant density peaking is obtained with increasing input power. This way physics parameters that lead to such increase in the density peaking shall be elucidated. While well-known candidates, such as the collisionality, previously found in other studies are also recovered in this study, it is furthermore found that edge $E \times B$ shearing may adopt a crucial role by enhancing the inward pinch. These results may indicate that a plasma with rotational shear could develop a stronger density peaking as compared to a non-rotating one, because its inward convection is increased compared to the outward diffusive particle flux as long as this rotation has a significant on $E \times B$ flow shear stabilisation. The possibly significant implications for future devices, which will exhibit much less torque compared to present day experiments, are discussed.
\end{abstract}

\section{INTRODUCTION}

In magnetic confinement fusion experiments, highly complex and nonlinear plasma turbulence determines the dominant fraction of cross-field heat, particles, and momentum transport. The performance of future fusion devices, for instance in terms of the confinement time, or the fusion power, are often estimated by means of empirical scaling laws. However, interplays between parameters like the local normalised pressure $\beta$, the collisionality, the safety factor $q$, or sheared $E \times B$ flow are known. Designing new promising operation scenarios as well as confidently extrapolating present-day knowledge to future devices thus requires a rigorous understanding of the underlying physics.

Significant advances have been achieved in recent years, many of them obtained through abinitio gyrokinetic simulations and mainly addressing the heat transport as subject of interest. The significant suppression of Ion Temperature Gradient (ITG) dominated plasma turbulence via electromagnetic fluctuations and fast ions [1, 2], for instance, has opened up the scope of turbulence reduction by alternative mechanisms to the wellknown $E \times B$ shearing. Such effects have also been shown to play an important role in the so-called isotope effect [3, 4, and the recently found $E \times B$ staircase phenomenon [5] 7 .

However, particle transport has received much less attention in ab-initio gyrokinetic simulations.
This is a significant drawback given that in comparison to the temperature profile,the impact of the different turbulence regimes in the density tends to be crucial because of the strong impact on the particle pinch, which changes direction depending on the main turbulence types. Therefore, the density can be peaked even in the case of no particle source and this is a fundamental difference with respect to the heat channel. This feature has been extensively studied in the framework of the linear or quasi-linear gyrokinetic theory [8 13] as well as from the experimental point of view [14-17]. In particular, the dependence of the pinch, outward or inward, on the turbulence regime, Trapped Electron Modes (TEM) or ITG and its dependence on other physical parameters, such as collisionality, has been highlighted as one of the main reasons for the change of density peaking in different plasma regimes. However, the question whether the physical mechanisms reducing heat transport, in particular those recently discovered, play a similar role in particle transport or particle pinch, still remains. The answer to this question is essential as accurate particle transport understanding and predictions are required since fusion power or bootstrap current, whose contribution are crucial for the future tokamak reactor, enormously depend on them.

In order to analyse what physical mechanisms play a role on particle confinement, and particularly on density peaking, JET discharges with a relatively weak energy confinement degradation 
and increased density peaking (at constant lineaveraged density) with input power [18] have been chosen for analysis. This work extends a previous study 19 with the gyrokinetic turbulence GENE 20], which was mainly focused on heat transport in the inner plasma region, by covering outer radial positions as well as adding the analysis of particle transport including pinch effects. It is often concluded from experiment and quasilinear models that a particle pinch exists, however, non-linear gyrokinetic simulations covering both the inner and outer part of the core plasma are still missing. The paper is organised as follows: the modelling set-up is discussed in section II, heat and particle transport at two radial locations is studied in section III, a review of previous particle transport analyses is given in section IV, a first analysis of particle transport is done in section $\mathrm{V}$ whereas the new interplay between inward particle pinch and $E \times B$ is shown in section VI. In section VII the conclusions are given.

\section{SIMULATION SETUP}

Two discharges are selected for the analysis of the JET-ILW power scan: 84798 at $6 \mathrm{MW}$ NBI power and 84792 at $13 \mathrm{MW}$ NBI power. For each of these, an interpretative power balance analysis with experimental data as input has been performed with the CRONOS suite of codes [21. This allows to determine the NBI power deposition profiles, the magnetic geometry, as well as the power balance turbulent heat fluxes $Q_{s}$ and particle fluxes $\Gamma_{s}$, which are consistent with the calculated source profiles of each species $s$. The fluxes are averaged over a sufficient time window $t=45.2 \mathrm{~s}-45.45 \mathrm{~s}$ at a given flux surface. In a second step, these fluxes are compared with the ones obtained from nonlinear GENE simulations. The work at hand extends previous studies documented in Ref. [19] by the analysis of particle transport and density peaking. Furthermore, GENE simulations at the radial position $\rho_{\text {tor }}=0.65$ (outer core) are added to 0.33 (inner core) results. We make use of the local (flux-tube) version of GENE, taking up to three plasma species (electrons and thermal + NBI fast ions) into account. Dedicated tests have demonstrated that adding impurity species $(\mathrm{C}, \mathrm{Be}, \mathrm{W})$ only marginally affects the results. At least $\{192,96,24,32,12\}$ grid cells in $\left\{x, y, z, v_{\|}, \mu\right\}$ phase space are employed in the nonlinear GENE simulations. Only ion scales, with $k_{y, \max }=1.2$ were analysed. Several numerical convergence tests have been performed in all dimensions by significantly increasing the box resolution for selected runs and comparing the results with the standard box used in the paper.

\begin{tabular}{ccccc}
\hline \hline & $\rho=0.33$ & \multicolumn{3}{c}{$\rho=0.65$} \\
& $6 \mathrm{MW}$ & $13 \mathrm{MW}$ & $6 \mathrm{MW}$ & $13 \mathrm{MW}$ \\
\hline$\nu_{i}^{\star}$ & 0.04 & 0.01 & 0.09 & 0.02 \\
$1 / \rho^{\star}$ & 300.72 & 242.41 & 432.69 & 343.07 \\
$\beta_{e}[\%]$ & 1.26 & 2.00 & 0.50 & 0.74 \\
$q$ & 0.98 & 0.92 & 1.67 & 1.55 \\
$\hat{s}$ & 0.30 & 0.18 & 1.45 & 1.71 \\
$R / \mathrm{m}$ & 3.00 & 3.03 & 3.00 & 3.03 \\
$\alpha_{\mathrm{MHD}}$ & 0.13 & 0.27 & 0.24 & 0.35 \\
$\gamma_{E \times B} /\left(c_{s} / R\right)$ & 0.19 & 0.23 & 0.36 & 0.53 \\
$Z_{\mathrm{eff}}$ & 1.16 & 1.26 & 1.16 & 1.31 \\
$T_{0 e} / \mathrm{keV}$ & 2.08 & 3.17 & 1.01 & 1.58 \\
$n_{0 e} / \mathrm{m}^{3}$ & 4.37 & 4.35 & 3.59 & 3.21 \\
$R / L_{T i}$ & 4.53 & 5.65 & 6.21 & 5.65 \\
$R / L_{T e}$ & 4.53 & 4.07 & 6.21 & 5.59 \\
$R / L_{n e}$ & 0.69 & 1.54 & 2.50 & 3.44 \\
$\left\langle Q_{i} V^{\prime}\right\rangle / \mathrm{MW}$ & 1.02 & 2.86 & 2.36 & 5.99 \\
$\left\langle Q_{e} V^{\prime}\right\rangle / \mathrm{MW}$ & 0.49 & 1.40 & 1.60 & 4.47 \\
\hline \hline
\end{tabular}

Table I. Local parameters obtained from CRONOS for the 6 and 13MW NBI power depositions in the JET power scan at low triangularity $\delta$. A finer reevaluation of the CRONOS interpretative simulations was performed compared to Ref. 19.

For gaining insights into relevant physics in these inner and outer core cases, parameters are varied around the experimental operation point. The nominal parameters (Tab. I) are extracted from interpretative CRONOS simulations. Some choices made in this procedure are detailed in the following. In Ref. 19, a q-profile sensitivity study has been performed, considering magnetic equilibria with $q=1.24$ and $q=0.92$ at $\rho_{\text {tor }}=0.33$, respectively. Here, only the $q=0.92$.

The measured $T_{i}$ from Charge Exchange (CX) for the low power discharge is slightly below $T_{e}$. This might not be accurate due to the relatively high collisionality and similar ion and electron heating in this plasma. Taking into account the $T_{i}$ error bars from the CX measurements, $T_{i}=T_{e}$ would be possible, as well. This assumption leads to significantly better agreement with the total plasma energy content when analyzed with the CRONOS code. In the following, $T_{i}=T_{e}$ is therefore assumed for the discharge 84798. Uncertainties exist also in the plasma rotation and the rate of flow shear, to which the turbulence level is very sensitive, particularly at outer radii. In any case, the sensitivity of the results obtained in the following sections to these parameters will be analysed by performing corresponding scans. 

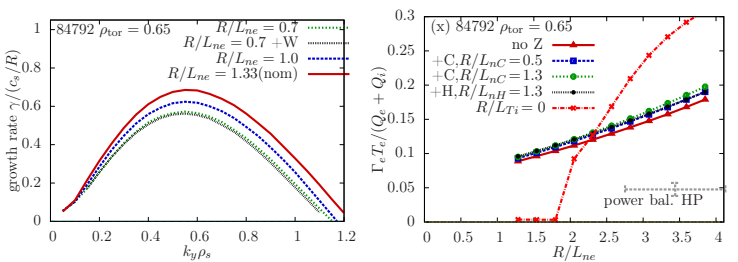

Figure 1. Growth rate spectra (a) and (b) the flux ratio at $k_{y} \rho_{s}=0.33$ as function of $R / L_{n e}$ for various gradient variations of the $13 \mathrm{MW}$ discharge at $\rho=0.65$.

\section{GENE RESULTS AND POWER BALANCE}

In Ref. 19 it was shown that at $\rho_{\text {tor }}=0.33$, both discharges are in the ion temperature gradient (ITG) driven regime, in which electromagnetic stabilisation plays a significant role at high heating power. Extending this study to the more outward radial position $\rho_{\text {tor }}=0.65$, we find that Density Gradient Trapped Electron Modes (DGTEM) highly contribute to the mode growth rate as depicted in Fig. 11(a) for several $R / L_{n e}$ scans for the $13 \mathrm{MW}$ discharge. The ratio of particle to quasilinear heat flux $\Gamma_{e} T_{e} / Q$ is also affected by the DGTEM being this impact particularly strong at artificially suppressed ITG drive $R / L_{T i}=0$ as depicted in Fig. 1(b).

Therefore, this plasma is found in an ITG+TEM regime due to the relatively high density peaking or gradient, respectively. The reliable determination of density gradients in experiments is therefore essential for a correct flux evaluation. Considering impurities (Hydrogen, Carbon, or Beryllium) with $Z_{\text {eff }} \lesssim 1.3$ and various density gradients can somewhat change the growth rates, but the ratio $\Gamma_{e} T_{e} / Q$ is not affected.

The power through a given flux surface $\left\langle Q_{s}\right\rangle V^{\prime}$ as obtained from CRONOS roughly increases proportional to the input power, as can be seen in Fig. 2. Assuming a Gyro-Bohm-like turbulent transport scaling $Q \sim Q_{g B}=c_{s} p_{e}\left(\rho_{s} / R\right)^{2} T^{5 / 2}$, doubling the input power would result in $2^{2 / 5}=$ 1.3 higher temperature and almost unchanged logarithmic temperature gradient $R / L_{T}=-R \nabla T / T$ if the transport stiffness is preserved. Here, the sound velocity $c_{s}=\left(T_{e} / m_{D}\right)^{1 / 2}$ and the gyroradius $\rho_{s}=c_{s} \Omega_{i}$ are defined, with cyclotron frequency $\Omega_{i}=m_{D} c /\left(e B_{0}\right)$ and $p_{e}$ denotes the electron pressure. However, in the present scan, the temperature increases by a significantly larger factor and appears to be more peaked in the core, see Refs. [18, 19. This implies that the fluxgradient relation $Q\left(R / L_{T}\right)$ is modified by additional physics, such as electromagnetic $(\beta$ - $)$ effects, equilibrium $E \times B$ flow shear, increased $T_{i} / T_{e}$, or the presence of fast ions. In the present set of
JET experiments, all of the mentioned effects simultaneously increase with NBI beam power. Theoretical analyses are required to disentangle their impact, and thus establish more confident extrapolations to higher heating power and future devices, in which the relation between relevant parameters will be different.

As depicted in Fig 2 and Fig 3, the CRONOS heat fluxes can be largely matched by GENE within the error bars of the logarithmic ion temperature gradient $R / L_{T i}$ which represents the main driving gradient at both radial positions. However, the ratio of the particle-to-heat-flux $\Gamma_{e} T_{e} / Q$ is found to be significantly larger in GENE than in CRONOS, especially at the outer radial position. The trend of higher $\Gamma_{e} T_{e} / Q$ at higher power is captured, though. Interestingly, the ratio $\Gamma_{e} T_{e} / Q$ decreases with $R / L_{T}$ for both discharges showing that the thermodiffusion pinch might be negative. This will be further discussed in section V. Generally, the discrepancies between GENE and CRONOS may be due to input uncertainties. The fact that such discrepancies increase towards the edge may be evidence for associated uncertainties in the evaluation of the interpreted particle flux in that region. The latter may be less accurate if particle sources or sinks - for instance, by means of gas injection or Edge Localized Modes (ELMs) - play a role at outer radial position but are not considered in the CRONOS simulations. This will be analysed in the future. Before further parameter scans in linear and nonlinear simulations are presented in Sec. V] it is useful to briefly review the present knowledge concerning particle fluxes in fusion plasmas.

\section{PARTICLE FLUX IN GYROKINETICS}

A rather complete overview in this context is given by Angioni and co-workers 912 who showed the strong impact of collisionality on the particle flux through a change of the particle pinch. Most of the previous studies have, however, been performed in the electrostatic limit $(\beta \rightarrow 0)$. Also more recent nonlinear simulation work falls into this regime 22]. An early extension to the electromagnetic regime is presented in Ref. 23, where increased particle flux due to larger $\beta$ is found. This effect can be mainly related to the non-adiabaticity of passing electrons and it is partially balanced by considering a self-consistent equilibrium pressure gradient $\left(\alpha_{\mathrm{MHD}}\right)$. The above reference furthermore confirms the validity of the collisionality dependence of the ITG caused outward particle flux in the electromagnetic regime. However, the direct applicability of such studies to NBI heated plasmas is difficult as a higher $\beta$ is obtained while simulta- 

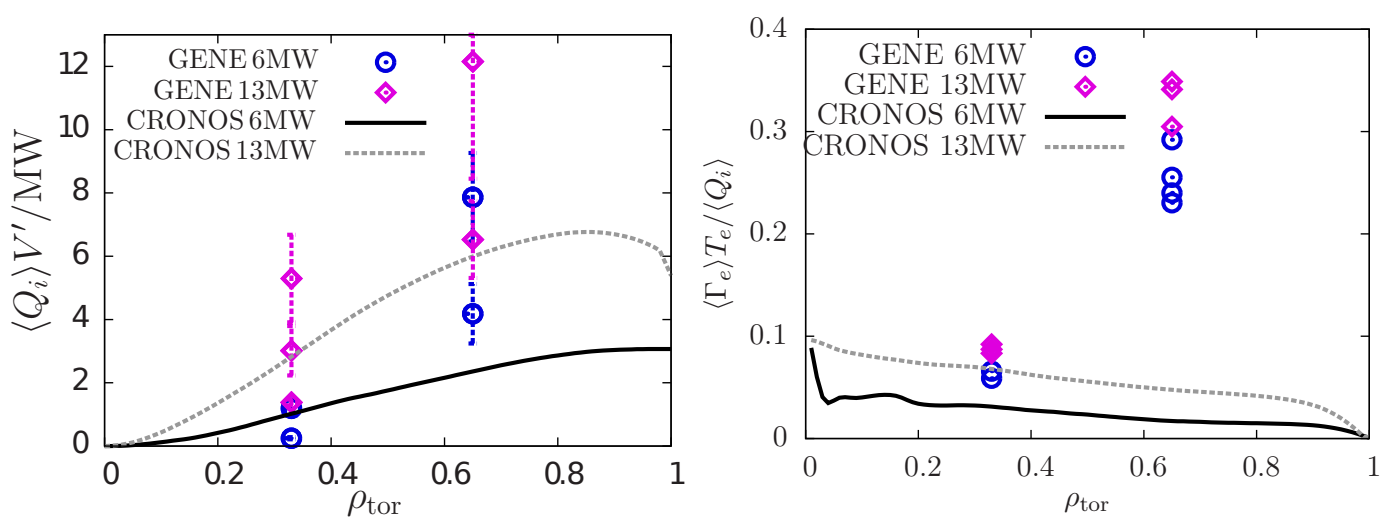

Figure 2. (a) Comparison between the power balance ion heat flux obtained from CRONOS and the fluxes obtained with GENE non-linear simulations (data from $R / L_{T i}$ scans of Fig. 3). (b) Comparison between $\Gamma_{e} T_{e} / Q_{i}$ calculated from CRONOS and the values obtained from GENE simulations.
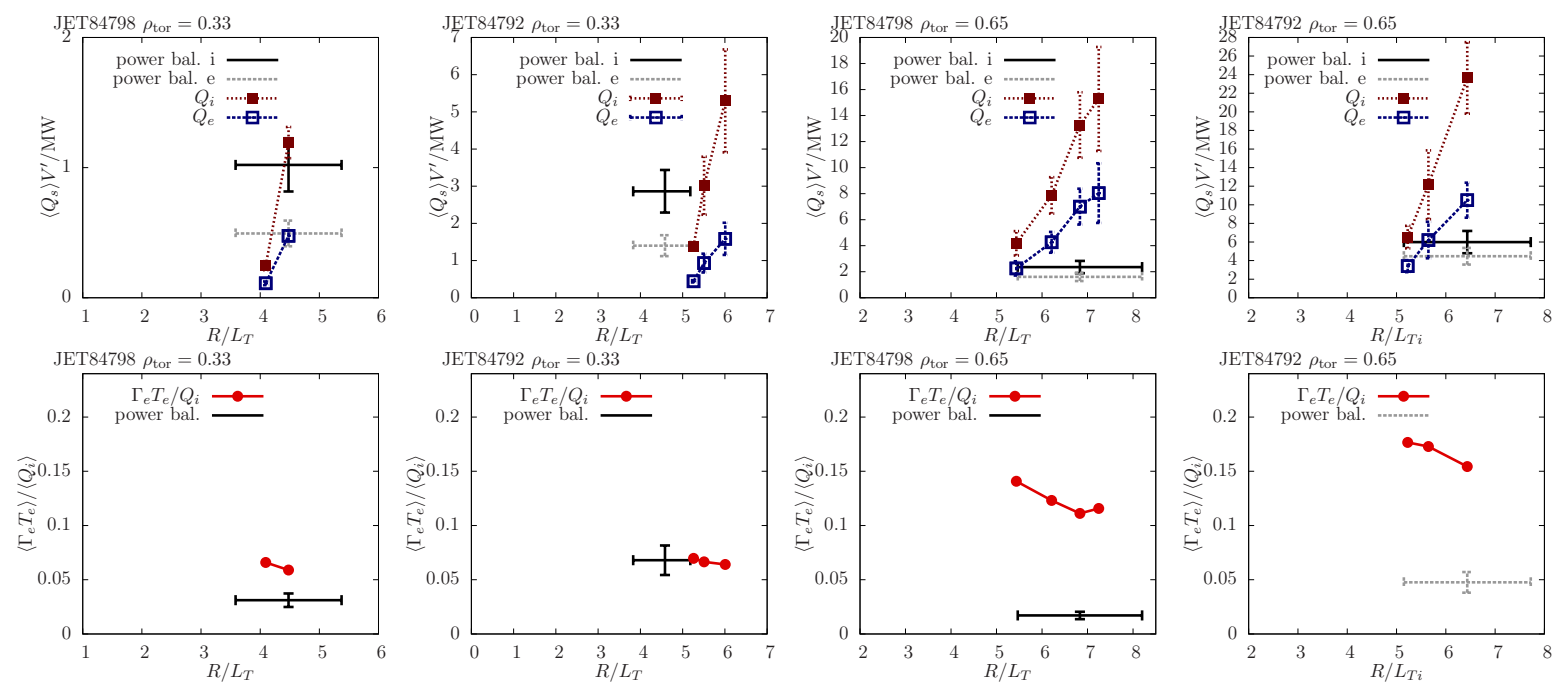

Figure 3. Same simulation data as in Fig: 2 $R / L_{T i}$ scans for the $6 \mathrm{MW}(84798)$ and $13 \mathrm{MW}(84792)$ discharges at two radial positions.

neously increased particle source, which is known to play a role on the density peaking [17, 24, 27. and that may counteract $\beta$-enhanced fluxes. Additionally, the NBI tend to increase the torque and plasma rotation which can have significant impact on the shearing flows. At least in the ITG/TEM mixed turbulence regime, sheared flows can indirectly change the balance between those two types of turbulence, since they more strongly affect ion modes. The direct impact of the rotation terms on the particle flux has been studied in Ref. [28, 29]. In an ITG example, Deuterium particle transport is found to be dominated by the convective flux $\left(C_{p}\right)$, which is hardly affected by plasma rotation. The roto-diffusion coefficient $\left(C_{u}\right)$ is small and inward. However, the dependence of the particle flux on flow shear has not been intensively studied, partially because it is not easily accessible with the quasi-linear approach. Such analysis is specially important in highly electromagnetic regimes as the aforementioned JET power scan in order to disentangle the impact of different effects collinear with NBI heating.

\section{PARAMETER SCANS IN NONLINEAR SIMULATIONS}

In the following, we perform nonlinear parameters scans to assess the scaling of turbulent transport with focus on the 13MW high power discharge with high density peaking. In line with expectation, lower collisionality and reduced density gradient are found to yield lower $\Gamma_{e} T_{e} / Q_{i, e}$. Offdiagonal terms play a role as well: thermodiffusion tends to be inward as could already be concluded from the $R / L_{T i}$ scans in Fig. 3 . Increased $\beta$ has no significant impact on $\Gamma_{e} T_{e} / Q i, e$, in contrast to the 
ASDEX Upgrade hybrid case discussed in Ref. 23]. While flow shear is very efficient in quenching the turbulence at outer radii, it has no strong impact on the $\Gamma_{e} T_{e} / Q_{i, e}$ ratio at $\rho_{\text {tor }}=0.65$. However, its impact is significantly increased at outer radii as will be demonstrated for $\rho_{\text {tor }}=0.75$ where a strong pinch reversal is observed.

\section{A. Collisionality scans}

Reaching lower collisionality can strongly increase the turbulence level and somewhat lower the $\Gamma_{e} T_{e} / Q_{i}$ flux ratio, as can be seen in Fig. 4 . In the 13MW discharge the collisionality is about a factor of two lower than in the 6MW discharge. Taking the parameters at $\rho_{\text {tor }}=0.65$ in the $13 \mathrm{MW}$ plasma and doubling the collisionality completely suppresses the turbulence because the stabilising factors $\beta$ and $\gamma_{E \times B}$ are stronger than in the low power case. In the other limit of $\nu_{e i}=0, \Gamma_{e} T_{e} / Q_{i}$ is about $25 \%$ smaller than the $6 \mathrm{MW}$ discharge at $\rho_{\text {tor }}=0.65$ and more than $50 \%$ at the inner radial positions. In these nonlinear simulations, the transport fluxes peak around $k_{y} \sim 0.35$ $\left(\rho_{\text {tor }}=0.33\right)$ and $k_{y}=0.4\left(\rho_{\text {tor }}=0.65\right)$ and radially outward particle flux is observed at all considered $k_{y}$ wavenumbers - regardless of the collision rate. Altogether, the stronger impact of collisionality on particle than on heat transport is recovered here.
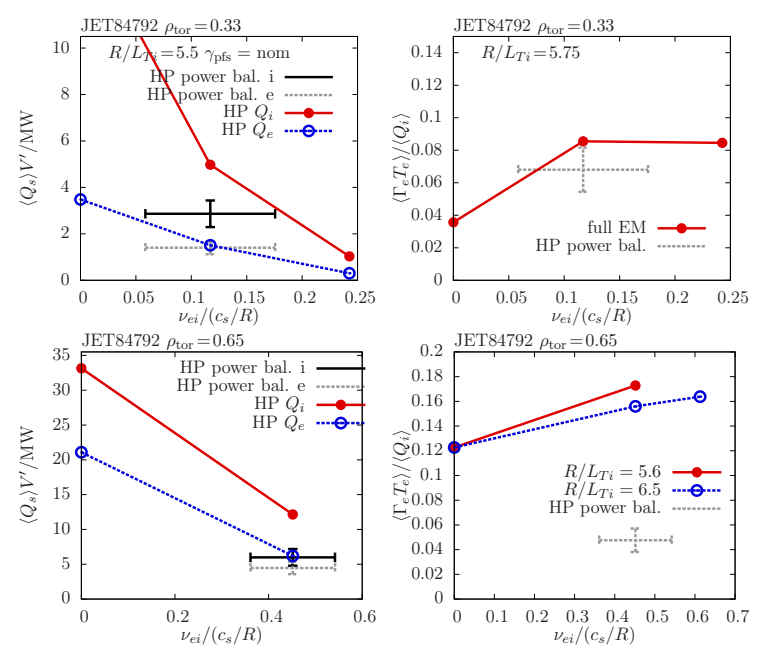

Figure 4. Impact of collisionality on the heat flux and the flux ratio in the $13 \mathrm{MW}$ high power discharge (84792) at $\rho=0.33\left(R / L_{T i}=5.5\right)$ and $\rho_{\text {tor }}=0.65$.

\section{B. Electromagnetic effects, $\beta$}

Regarding the impact of finite $\beta$, a strong turbulence stabilisation can be observed at the inner ra- dial position in the high power case while $\beta$ seems to play a less important role at $\rho_{\text {tor }}=0.65$. The linear simulations shown in Fig. 5 confirm the expected increase of $\Gamma_{e} T_{e} / Q_{i}$. In the nonlinear simulations, however, the reduction of the overall turbulence level is found, but $\Gamma_{e} T_{e} / Q_{i}$ remains basically unchanged.
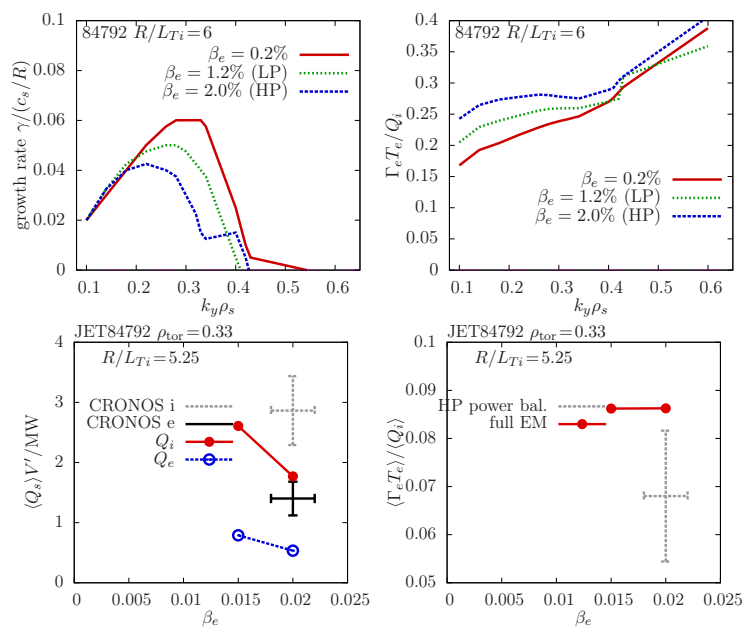

Figure 5. Impact of beta on (a) linear growth rate, (b) quasilinear ratio $\Gamma_{e} T_{e} / Q_{i}$, (c) nonlinear heat flux and (d) flux ratio for the $13 \mathrm{MW}$ discharge at $\rho=0.33$, fixing $R / L_{T i}=5.25$.

\section{Density gradient $R / L_{n e}$ variations}

Although the heat fluxes at $\rho_{\text {tor }}=0.33$ are found to respond non-monotonically to dedicated scans in the electron density gradient $R / L_{n e}$, the ratio $\Gamma_{e} T_{e} / Q_{i}$ clearly increases, as can be seen in Fig. 6 The figure furthermore demonstrates a similar behaviour at the $\rho_{\text {tor }}=0.65$ position. Often, the peaking factor is computed by matching the outward flux with the particle source by only scanning $R / L_{n}$, without considering other physics input. This procedure would predict a significantly more flat density peaking.

\section{Flow shear impact}

To our knowledge, the literature is short of nonlinear gyrokinetic simulations with particular focus on the impact of flow shear on particle confinement. In this paper, we therefore aim to fill the gap by performing non-linear gyrokinetic scans on the $E \times B$ shear, which is known to stabilize turbulence, but as well on the parallel velocity gradient (PVG) shear, which is known to be destabilizing in certain conditions. For that purpose, both corresponding shearing rate code inputs are varied. 

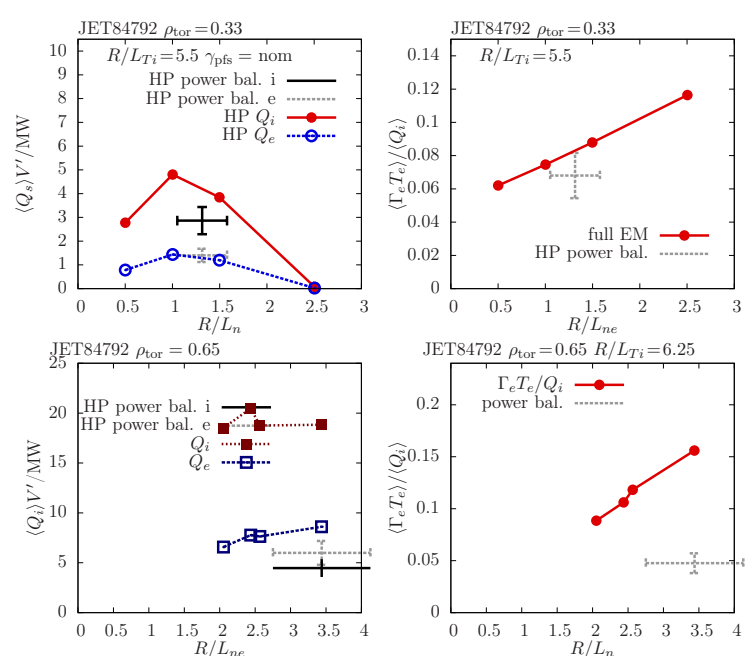

Figure 6. Impact of $R / L_{n e}$ on the heat flux and the flux ratio at $\rho=0.33\left(\left(R / L_{T i}=5.5\right)\right)$ and $\rho_{\text {tor }}=0.65$.

In the limit of strong equilibrium flows, PVG and $E \times B$ flow shear must be strictly related by the geometry of the magnetic field to ensure that the equilibrium flow is toroidal. Independent scans in PVG and $E \times B$ shear (with the other held fixed) break this constraint, but have been performed to expose the individual impacts of PVG and EXB shear on particle transport and the particle pinch.

As shown in Fig. 7, in the electromagnetic cases at $\rho_{\text {tor }}=0.33, E \times B$ shear reduces the heat flux only at values somewhat larger than the nominal one. The particle flux per heat flux remains almost unchanged. At $\rho_{\text {tor }}=0.65$ the overall flow shear stabilisation is more pronounced, in line with previous research 30, but $\Gamma_{e} T_{e} / Q_{i}$ is still not strongly affected (a slight reduction can be noted). For these $\rho=0.65$ cases, we show also the result for keeping $\gamma_{\mathrm{pfs}}=\gamma_{E \times B}$ in the scan, which leads to reduced stabilisation in line with 31, but does not alter $\Gamma_{e} T_{e} / Q_{i}$. However, in order to develop a better idea on the role of $E \times B$ on density peaking, a more extended study on its impact on the particle pinch has to be carried out. This will be done in the next section. Additionally, the radial location $\rho=0.75$ will be considered.

\section{PARTICLE PINCH IN NONLINEAR GYROKINETICS}

Some possible indications concerning the impact of $E \times B$ on particle transport and density peaking have been suggested both from modeling [26] and experiments 32 . However, so far no clear picture regarding the nature of such process could be drawn. Particle transport can be separated into a diffusive part $\Gamma^{D}=n D R / L_{n}$ and a non-diffusive particle part, $\Gamma^{V}$ as

$$
\Gamma_{e}=\Gamma^{D}+\Gamma^{V}=n_{e} D_{n e} \frac{a}{L_{n e}}+n_{e} V
$$

with $n_{e} V$ denoting a convective contribution with $V$ the particle pinch. However, the diffusive part can have an extra term from the temperature gradient drive (thermodiffusion). The final flow can be therefore decomposed as

$$
\Gamma_{e}=n_{e} D_{n e} \frac{a}{L_{n e}}+n_{e} D_{T e} \frac{a}{L_{T e}}+n_{e} V
$$

In this paper, an approximation to the ratio of pinch to diffusion is obtained by adding a passive electron species with $a / L_{n}=0$, which removes pure particle diffusive transport and yields the particle flux $\Gamma^{V}$. We expect that this approach of determining $\Gamma^{V}$ and $\Gamma^{D}$ captures the main pinch physics although the coefficients $V$ and $D$ of active electrons would depend on the density gradient to some extent.

In a first step, the particle flux separation as described in Eq. 1 shall now be employed to results at more outer radial positions where $E \times B$ shear effects tends to be even more relevant. The simulations here are performed for the $13 \mathrm{MW}$ case with $R / L_{n e}=1.72$. Fig. 8 displays the dependence of the ratio $\Gamma_{e} T_{e} / Q_{i}$ on the $E \times B$ shear at $\rho=0.75$ in the case of a pure $\gamma_{E \times B}$ scan and in the alternative case with $\gamma_{P V G}$ accordingly changed with the $E \times B$ shearing. Clearly and unlike the case at $\rho=0.65$ the $E \times B$ shearing has a strong effect on the ratio $\Gamma_{e} T_{e} / Q_{i}$ leading to $\Gamma_{e} T_{e} / Q_{i} \sim 0$ for $\gamma_{E \times B} / \gamma_{E \times B, \exp }=1.4$. Such trend is reduced when the PVG is included, which prevents the full stabilization of the particle flux.

The corresponding diffusive and convective fluxes spectra are analyzed in Fig. 9. For the diffusive part, $\Gamma^{D}$, the $E \times B$ shearing acts as expected, shifting the spectrum towards higher $k_{y} \rho_{s}$ (and therefore reducing the global transport) compared to the case with no $E \times B$ and PVG effects. The cases with only PVG or nominal $E \times B$ and PVG are in between these two extreme cases. Compared to this expected behavior, the convective flux $\Gamma^{V}$ has some unexpected features. Comparing the cases with no $E \times B$ and PVG and the case with only $E \times B$ we also observe a shift towards higher $k_{y} \rho_{s}$ but this shift is accompanied by a general reversal of the flux, which becomes significantly negative (therefore of inward pinch nature). The effect of PVG is found to be complex. It decreases the negative part of the spectrum when added to the simulations with only $E \times B$ or with no effects. However, since the positive part is also affected, the total pinch contribution can change from positive to negative even compared to the case without 

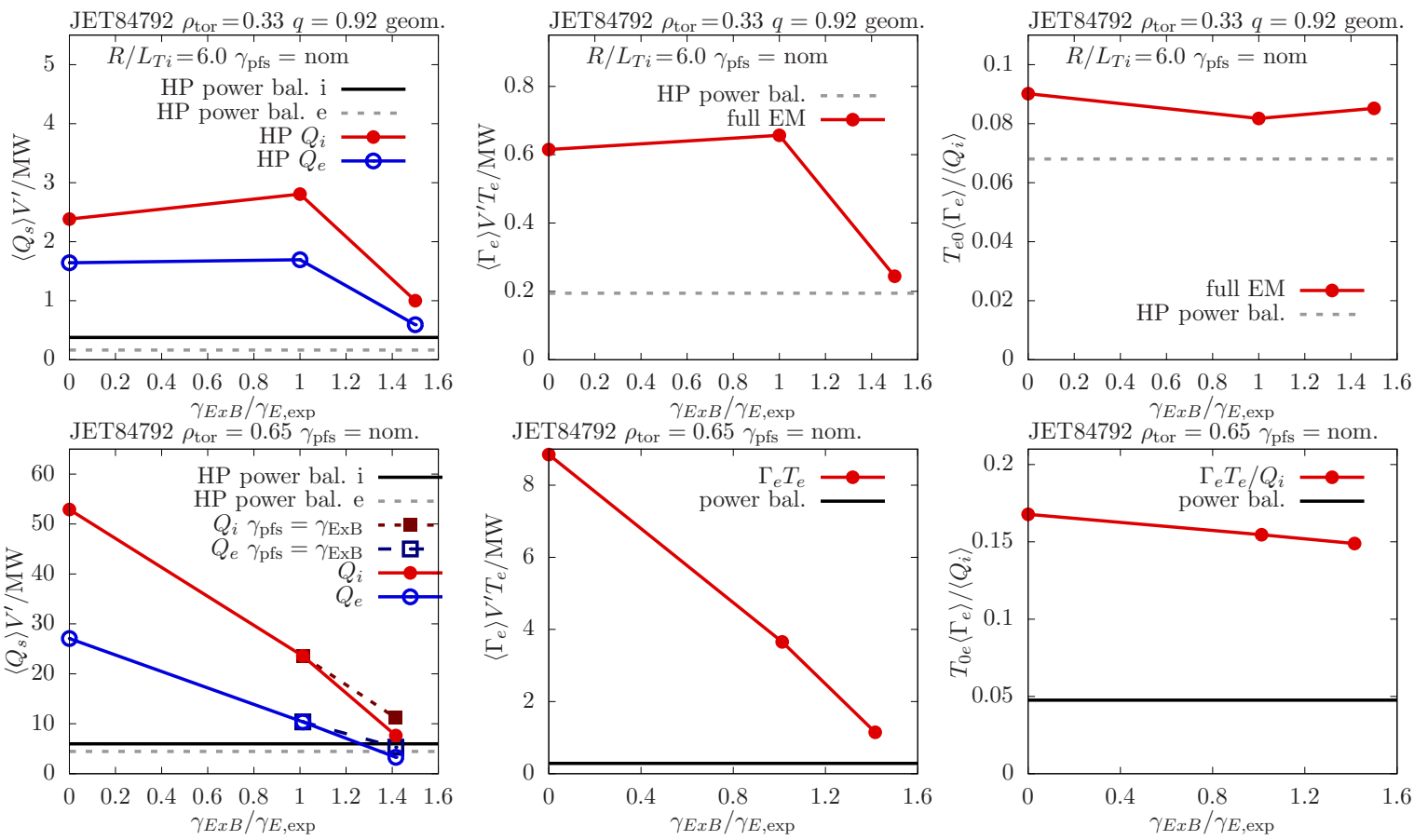

Figure 7. Impact of $E \times B$ rotation shear on heat flux (left), particle flux (center), and their ratio (right) for the $q=0.92$ geometry at $\rho_{\text {tor }}=0.33$ (top) and 0.65 (bottom). The CRONOS values are added as black-solid or gray-dashed lines for reference.

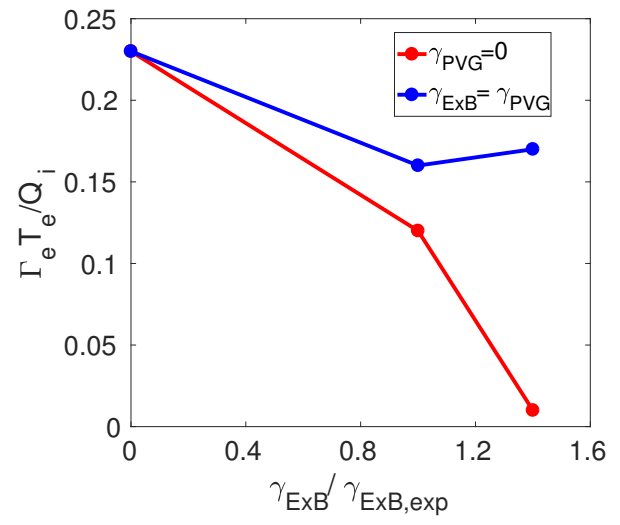

Figure 8. $\Gamma_{e} T_{e} / Q_{i}$ for the case with only $E \times B$ shearing $\gamma_{P V G}=0$ and with equal $E \times B$ and PVG shearing $\gamma_{E \times B}=\gamma_{P V G}$ at $\rho_{\text {tor }}=0.75$.

effects, as shown in Tab. II], where a summary of the changes on $\Gamma^{D}$ and $\Gamma^{V}$ is presented.

The particle pinch becomes strongly inward with increasing $E \times B$ shearing. This fact leads to a stronger impact of $E \times B$ shearing on the particle than the heat transport as demonstrated by means of the factor $\mathrm{RV} / \chi_{i}$ in Tab. II, although the different impact of $E \times B$ between particle and heat channels would depend on the turbulence level, i.e. on $R / L_{T i}$.

The density peaking would indeed benefit from

\begin{tabular}{cccc}
\hline \hline & $\mathrm{V}(\mathrm{m} / \mathrm{s})$ & $\mathrm{RV} / \chi_{i}$ & $\Gamma^{V} / \Gamma^{D}$ \\
\hline$E \times B=0 \mathrm{PVG}=0$ & 1.29 & 0.09 & 0.12 \\
$E \times B=0 \mathrm{PVG}=\mathrm{nom}$ & -0.49 & -0.12 & -0.15 \\
$E \times B=$ nom $\mathrm{PVG}=$ nom & -0.75 & -0.24 & -0.29 \\
$E \times B=$ nom $\mathrm{PVG}=0$ & -2.19 & -0.42 & -0.5 \\
\hline
\end{tabular}

Table II. Non-linear simulation results for different combinations of $E \times B$ and PVG values including nominal values (refereed as nom) with $\chi_{i}$ the ion heat diffusivity.

such trend as can be derived from the ratio $\Gamma^{V} / \Gamma^{D}$ which increases in absolute value and becomes negative from the case without to the case with $E \times B$ shearing. The role of PVG is complex as it can be stabilizing or destabilizing depending on the plasma conditions. Nevertheless, in nominal conditions for both $E \times B$ and $\mathrm{PVG}$, the pinch is inward and the ratio $\Gamma^{V} / \Gamma^{D}$ clearly shows that flow shearing plays a role on the increased density peaking obtained in those JET plasmas.

In order to shed light on the origin of the strong inward pinch with $E \times B$ shearing an alternative simulation has been performed by removing the thermodiffusion part of the pinch, i.e. by setting $L_{T e}=0$. The spectra for the cases with and without thermodiffusion contribution for the simulation with solely $E \times B$ shear but no PVG are displayed in Fig. 10. Clearly, the strong inward pinch 

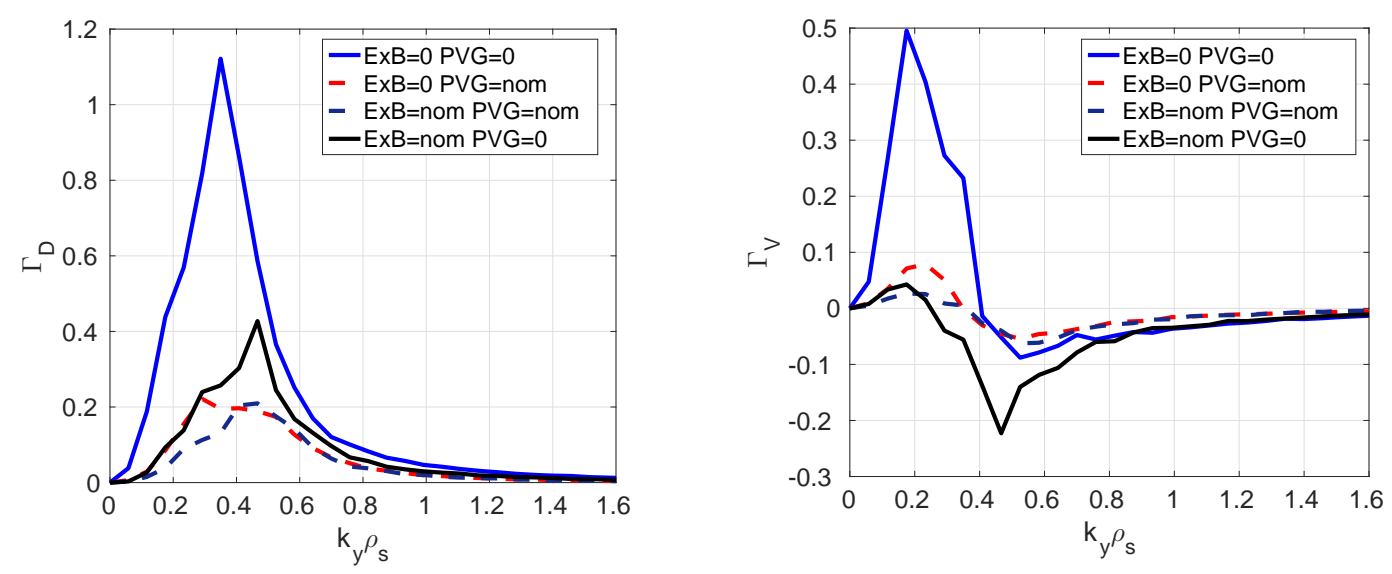

Figure 9. $\Gamma^{D}$ and $\Gamma^{V}$ spectrum for different combinations of $E \times B$ and PVG flow shearing at $\rho_{\text {tor }}=0.75$.

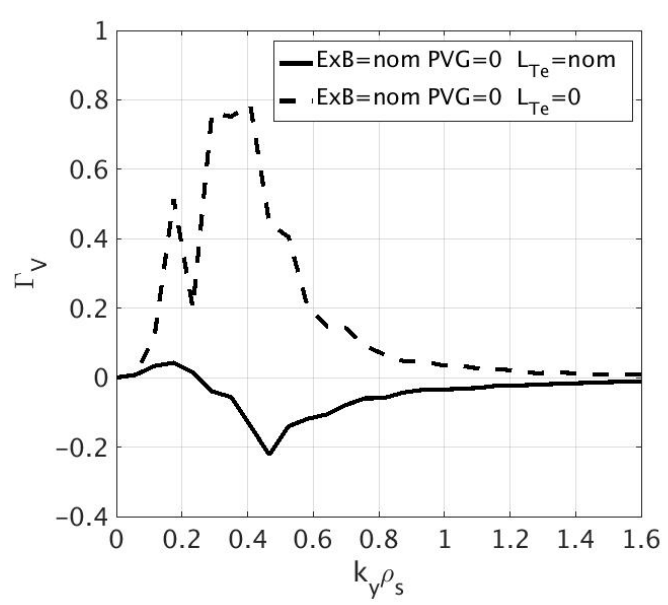

Figure 10. $\Gamma^{V}$ spectrum comparison between the case with only nominal $E \times B$ shearing and nominal $L_{T e}$ or $L_{T e}=0$ at $\rho_{\text {tor }}=0.75$.

with $E \times B$ shearing disappears if thermodiffusion is removed, implying that, as it happens with collisionality, the change of direction of the particle pinch with $E \times B$ shearing is also linked to thermodiffusion. This has profound implications on the way experiments could be planned as a way to study the dependence of density peaking with collisionality is to use NBI heating, which in addition to the fuelling leads to an extra collinear effect by increasing the toroidal rotation (the main source of $E \times B$ shearing). Decoupling such effects is essential for the extrapolation of present-days tokamak devices to futures ones as ITER, which will have presumably less toroidal rotation due to the weaker NBI torque.

\section{SUMMARY AND CONCLUSIONS}

The particle transport in JET plasmas with a significant increase of density peaking with NBI power has been studied by means of linear and non-linear gyrokinetic simulations.

Linear simulations show that these plasmas are ITG dominated and no transition to TEM is observed when the NBI power is changed although subdominant TEM are present due to the high density peaking.

From non-linear simulations it has been shown that while well established parameters playing a role on particle transport and density peaking could be recovered with the GENE simulations, some new factors have also been identified to be important. In particular, $E \times B$ flow shearing can enhance the inward particle pinch leading to a significant decrease of the ratio $\Gamma_{e} T_{e} / Q_{i}$. This effect is stronger at outer radial locations which means that it can be particularly significant close to the pedestal region. Therefore, in the outer part of the plasma, $E \times B$ shearing may have a stronger impact on particle than on heat transport although the final impact will depend on the competing effects of $E \times B$ and PVG flow shear.

These findings are further substantiated by an analysis of the ratio $\Gamma^{V} / \Gamma^{D}$ which demonstrates an enhanced inward pinch with $E \times B$ shearing. The latter may, for instance, be found to be significant in the outer part of rotating plasmas and therefore cause visible density peaking in such scenarios. On the other hand, it is also found that PVG can limit the inward pinch generated by the $E \times B$ shearing to some degree.

The results shown in this paper may have profound consequences on the way density peaking is understood. Whereas the dependence on collisionality has been highlighted as the main cause for 
generating a particle pinch, the experimental evidence leading to such conclusion were based in a non-negligible part on NBI-heated plasmas where the NBI can collinearly increase the plasma torque and therefore change the $E \times B$ shearing. This suggests a need for new experiments to isolate the independent impacts of collisionality and rotational shearing on density peaking. This could lead as well to the change of some scaling laws used for the extrapolation of present day experiments. This is an essential step towards the proper evaluation of density peaking in future tokamak devices with different plasma conditions.

An interesting discussion is whether this phenomenon is exclusive to JET. Based on the analysis shown in this paper, one may conclude that as long as a plasma is in ITG dominated turbulence with a relatively strong $E \times B$ shearing compared to turbulence growth rates, the same phenomenon can play a role at the plasma edge. This has been shown to be the case even for ITER with self-consistent TGLF simulations, where the density has a significant increasing of peaking due to the inclusion of $E \times B$ flow shear. Such increasing starts at the edge, next to the pedestal 33 .

However some drawbacks have also been encountered in this study. In particular, it remains difficult to match the source balance particle flux in the outer part of the plasma which leads to a overestimation of the ratio $\Gamma_{e} T_{e} / Q_{i}$. This shows that the proper evaluation of the particle sources and sinks in that plasma region is essential.

Further rotating frame effects like centrifugal and coriolis drifts have not been considered here, and may be candidates for some extra missing physics ingredients.

\section{ACKNOWLEDGEMENTS}

The authors would like to thank C. Angioni, A. Di Siena, E. Poli, M. J. Pueschel and D. Told for valuable discussions. This work has been carried out within the framework of the EUROfusion Consortium and has received funding from the Euratom research and training programme 2014-2018 and 2019-2020 under grant agreement No 633053 . The views and opinions expressed herein do not necessarily reflect those of the European Commission.

The numerical results presented in this work were carried out using the HELIOS supercomputer system at the Computational Simulation Centre of International Fusion Energy Research Centre (IFERC-CSC), Aomori, Japan, under the Broader Approach collaboration between Euratom and Japan, implemented by Fusion for Energy and JAEA, as well as using the resources of the MPCDF computing center, Garching, Germany, the BSC, Barcelona, Spain, under the PRACE project ZONALGENE and the MARCONI supercomputer system at CINECA, Italy.

\section{REFERENCES}

[1] J. Citrin, F. Jenko, P. Mantica, D. Told, C. Bourdelle, J. Garcia, J. W. Haverkort, G. M. D. Hogeweij, T. Johnson, and M. J. Pueschel, Phys. Rev. Lett. 111, 155001 (2013).

[2] J. Garcia, C. Challis, J. Citrin, H. Doerk, G. Giruzzi, T. Görler, F. Jenko, P. Maget, and JET Contributors, Nucl. Fusion 55, 053007 (2015).

[3] J. Garcia, T. Görler, F. Jenko, and G. Giruzzi, Nuclear Fusion 57, 014007 (2017).

[4] J. Garcia, T. Görler, and F. Jenko, Physics of Plasmas 25, 055902 (2018).

[5] G. Dif-Pradalier, G. Hornung, X. Garbet, P. Ghendrih, V. Grandgirard, G. Latu, and Y. Sarazin, Nuclear Fusion 57, 066026 (2017)

[6] W. Wang, Y. Kishimoto, K. Imadera, J. Li, and Z. Wang, Nuclear Fusion 58, 056005 (2018).

[7] L. Qi, J.-M. Kwon, T. Hahm, S. Yi, and M. Choi, Nuclear Fusion 59, 026013 (2019).

[8] C. Bourdelle, Plasma Physics and Controlled Fusion 47, A317 (2005)

[9] C. Angioni, E. Fable, M. Greenwald, M. Maslov,
A. G. Peeters, H. Takenaga, and H. Weisen, Plasma Phys. Controll. Fusion 51, 124017 (2009).

[10] E. Fable, C. Angioni, and O. Sauter, Plasma Phys. Controll. Fusion 52, 015007 (2010)

[11] C. Angioni, R. M. McDermott, F. J. Casson, E. Fable, A. Bottino, R. Dux, R. Fischer, Y. Podoba, T. Pütterich, F. Ryter, and E. Viezzer, Phys. Rev. Lett. 107, 215003 (2011).

[12] C. Angioni, Y. Camenen, F. J. Casson, E. Fable, R. M. McDermott, A. G. Peeters, and J. E. Rice, Nucl. Fusion 52, 114003 (2012)

[13] M. Romanelli, G. Regnoli, and C. Bourdelle, Physics of Plasmas 14, 082305 (2007)

[14] G. T. Hoang, C. Bourdelle, B. Pégourié, B. Schunke, J. F. Artaud, J. Bucalossi, F. Clairet, C. Fenzi-Bonizec, X. Garbet, C. Gil, R. Guirlet, F. Imbeaux, J. Lasalle, T. Loarer, C. Lowry, J. M. Travère, and E. Tsitrone (Tore Supra Team), Phys. Rev. Lett. 90, 155002 (2003)

[15] G. T. Hoang, C. Bourdelle, X. Garbet, J. F. Artaud, V. Basiuk, J. Bucalossi, F. Clairet, C. FenziBonizec, C. Gil, J. L. Ségui, J. M. Travère, 
E. Tsitrone, and L. Vermare, Phys. Rev. Lett. 93, 135003 (2004)

[16] C. Angioni, A. G. Peeters, G. V. Pereverzev, F. Ryter, and G. Tardini (ASDEX Upgrade Team), Phys. Rev. Lett. 90, 205003 (2003)

[17] H. Weisen, A. Zabolotsky, C. Angioni, I. Furno, X. Garbet, C. Giroud, H. Leggate, P. Mantica, D. Mazon, J. Weiland, L. Zabeo, K.-D. Zastrow, and J.-E. contributors, Nuclear Fusion 45, L1 (2005)

[18] C. Challis, J. Garcia, M. Beurskens, P. Buratti, E. Delabie, P. Drewelow, L. Frassinetti, C. Giroud, N. Hawkes, J. Hobirk, E. Joffrin, D. Keeling, D. King, C. Maggi, J. Mailloux, C. Marchetto, D. McDonald, I. Nunes, G. Pucella, S. Saarelma, J. Simpson, and JET Contributors, Nucl. Fusion 55, 053031 (2015).

[19] H. Doerk, C. Challis, J. Citrin, J. Garcia, T. Görler, F. Jenko, and J. Contributors, Plasma Phys. Controll. Fusion 58, 115005 (2016).

[20] W. Dorland, F. Jenko, M. Kotschenreuther, and B. N. Rogers, Phys. Rev. Lett. 85, 5579 (2000).

[21] J. Artaud, V. Basiuk, F. Imbeaux, M. Schneider, J. Garcia, G. Giruzzi, P. Huynh, T. Aniel, F. Albajar, J. Ané, A. Bécoulet, C. Bourdelle, A. Casati, L. Colas, J. Decker, R. Dumont, L. Eriksson, X. Garbet, R. Guirlet, P. Hertout, G. Hoang, W. Houlberg, G. Huysmans, E. Joffrin, S. Kim, F. Köchl, J. Lister, X. Litaudon, P. Maget, R. Masset, B. Pégourié, Y. Peysson, P. Thomas, E. Tsitrone, and F. Turco, Nucl. Fusion 50, 043001 (2010).

[22] D. R. Mikkelsen, M. Bitter, L. Delgado-Aparicio, K. W. Hill, M. Greenwald, N. T. Howard, J. W. Hughes, J. E. Rice, M. L. Reinke, Y. Podpaly, Y. Ma, J. Candy, and R. E. Waltz, Phys. Plasmas 22, $062301(2015)$
[23] T. Hein, C. Angioni, E. Fable, and J. Candy, Phys. Plasmas 17, 102309 (2010)

[24] H. Weisen, A. Zabolotsky, M. Maslov, M. Beurskens, C. Giroud, D. Mazon, and J.-E. contributors, Plasma Phys. Controll. Fusion 48, A457 (2006)

[25] M. Maslov, C. Angioni, H. Weisen, and JETEFDA contributors, Nucl. Fusion 49, 075037 (2009)

[26] J. Garcia, N. Hayashi, B. Baiocchi, G. Giruzzi, M. Honda, S. Ide, P. Maget, E. Narita, M. Schneider, H. Urano, and the JT-60U Team, Nuclear Fusion 54, 093010 (2014)

[27] T. Tala, in 27th IAEA Int. Conf. on Fusion Energy, $E X / 4-4$ (2018).

[28] F. J. Casson, A. G. Peeters, C. Angioni, Y. Camenen, W. A. Hornsby, A. P. Snodin, and G. Szepesi, Phys. Plasmas 17, 102305 (2010).

[29] F. J. Casson, A. G. Peeters, C. Angioni, Y. Camenen, W. A. Hornsby, A. P. Snodin, and G. Szepesi, Phys. Plasmas 19, 099902 (2012)

[30] J. Citrin, J. Garcia, T. Görler, F. Jenko, P. Mantica, D. Told, C. Bourdelle, D. R. Hatch, G. M. D. Hogeweij, T. Johnson, M. J. Pueschel, and M. Schneider, Plasma Phys. Control. Fusion 57, 014032 (2015).

[31] J. Citrin, F. Jenko, P. Mantica, D. Told, C. Bourdelle, R. Dumont, J. Garcia, J. W. Haverkort, G. M. D. Hogeweij, T. Johnson, M. J. Pueschel, and J.-E. contributors, Nucl. Fusion 54, 023008 (2014)

[32 X. Wang, S. Mordijck, L. Zeng, L. Schmitz, T. L. Rhodes, E. J. Doyle, R. Groebner, O. Meneghini, G. M. Staebler, and S. P. Smith, Plasma Physics and Controlled Fusion 58, 045026 (2016)

[33] C. Chrystal, in 27th IAEA Int. Conf. on Fusion Energy, EX/5-2 (2018). 